\title{
Tratamiento biológico de madera para eliminar pitch en la producción de celulosa
}

\author{
Cecilia Rodríguez \\ Hiram Medrano \\ Nuria Rocha \\ Alberto Gallegos 1 \\ Martha Rosales \\ Rubén González-Laredo
}

\section{RESUMEN}

\begin{abstract}
Se realizó un estudio biotecnológico para abatir el contenido resinoso de astilla de pino del estado de Durango y evitar los problemas asociados con la formación de pitch en la producción de pulpa mecánica. Se aislaron 49 cepas nativas que degradaron ácidos resínicos y precursores de pitch. Entre las mejores cepas se identificaron a Paecilomyces sp., Penicillum sp., Phialophora sp., Trichosporon sp., Rhodotorula sp., Cryptococcus sp. y Chryseomonas luteola. Esta última fue la más eficiente con un rendimiento de $87,3 \%$ a nivel matraz, por lo que fue probada preliminarmente en fermentación sólida tipo heap leaching. La cepa Paecilomyces sp. rindió $86,6 \%$ de efectividad a nivel matraz y fue probada en fermentación semisólida. Los resultados de las pruebas a nivel matraz fueron muy superiores a los obtenidos de manera preliminar en las fermentaciones sólidas.
\end{abstract}

PALABRAS CLAVE:

Biodegradación, extraíbles, fermentación, pitch, pulpa y papel.

\begin{abstract}
Durango, Mexico pine woods have higher resin content than those from southern US. For this reason, a biotechnological study to remove resinic acids and other pitch precursors in pine chips was performed to improve the production of a mechanical pulp mill. Forty nine native strains were isolated and some of them identified as Paecilomyces sp., Penicillum sp., Phialophora sp., Trichosporon sp., Rhodotorula sp., Cryptococcus sp. and Chryseomonas luteola. The later one was the most active in degrading resin components in the flask (87,3 $\%)$ and tested in preliminary heap leaching fermentation assays. Paecilomyces sp. was the second most effective $(86,6 \%)$ and tested in semisolid fermentation trials. Both solid phase fermentation processes afforded far lower yields compared to the flask experiments.
\end{abstract}

KEYWORDS:

Biodegradation, extractives, fermentation, pitch, pulp and paper. 


\section{INTRODUCCIÓN}

La madera es un material compuesto de celulosa, hemicelulosa, lignina y extraíbles que contienen material resinoso. En la producción de pulpa de madera, la presencia de esta resina es indeseable. La industria de la pulpa y papel se ve gravemente afectada por la presencia de resina, ya que ésta forma incrustaciones o precipitados difíciles de remover que genéricamente se denominan pitch (Back y Allen, 2000; Aguilar Rivera, 2004). La acumulación de pitch provoca problemas mecánicos y de mantenimiento en los equipos y líneas del proceso. Como consecuencia, se requiere remplazar con mayor frecuencia piezas dañadas, como platos de refinación, filtros, tuberías, etc., lo cual incrementa los períodos de tiempo muerto y los costos de producción (Lloyd et al., 1990).

El estado de Durango ocupa uno de los primeros lugares en la producción forestal mexicana. En la planta PIPSA-MEX S.A. de C.V. en la ciudad de Durango, se produce pulpa celulósica para la elaboración de papel y cartones con materias primas de la región (madera y astillas de pino), mediante el proceso BCTMP (proceso de pulpeo químico termomecánico blanqueado). El proceso y equipo con el que cuenta la empresa fueron originalmente diseñados para trabajar con pino del sur de Estados Unidos el cual, por razones climáticas, geográficas y botánicas, tiene $60 \%$ menos de resina que el pino promedio del bosque duranguense $y$ contienen extraíbles resinosos de 4 al $8 \%$ (Brush et al., 1994).

Los problemas provocados por la presencia del pitch en la empresa PIPSAMEX se traducen en altos costos de mantenimiento y continua sustitución de partes y equipo de proceso. Por ejemplo, para una producción aproximada de 4500 toneladas mensuales de pulpa se gastaban en el año 2001, \$128 000,00 en platos de refinación y \$26 000,00 en telas para la prensa lavadora. Estas cantidades son excesivas para la operación óptima y eficiente de una planta de esta naturaleza, por lo que se demandan soluciones para disminuir los costos asociados al pitch.
Adicionalmente, la presencia de la resina en la pulpa, provoca que el papel elaborado con ella sea de baja calidad y que no se alcance la blancura requerida, por lo que se requiere mayor intensidad en el tratamiento químico para lograrla. Los ácidos resínicos provocan en la celulosa un color amarillento y la formación de lunares indeseables. Cuando se requiere blanquear esta pulpa, para que pueda ser utilizada en la producción de papel, se necesita tratarla adicionalmente para reducir su contenido resinoso, cuando menos al $1 \%$. Este es el nivel máximo tolerado para cualquier uso, incluyendo la industria alimentaria (Mitsui et al., 1998). La aplicación de biotratamientos con cepas nativas es una opción viable que ha sido experimentada con relativos éxitos (Rocheleau et al., 1997).

\section{OBJETIVOS}

Disminuir el contenido resinoso precursor de pitch, en astilla de madera a un nivel aceptable para la producción de celulosa, antes de la refinación, aplicando un procedimiento biotecnológico.

Particularmente, aislar e identificar las cepas capaces de disminuir el contenido de resina en astillas de pino y realizar ensayos preliminares para llevar a cabo el proceso a nivel planta piloto.

\section{MATERIALES Y MÉTODOS}

1. La astilla de madera se muestreó según técnica TAPPI 257 cm-85 (TAPPI, 1985), asegurando representatividad de la materia prima con la que normalmente se abastece la planta. La astilla proviene de aserraderos de la región y la composición estimada de su origen es Pinus cooperi Blanco (35\%), Pinus durangensis Martínez (30\%), Pinus engelmannii Carr (12\%), Pinus arizonica Engelm (7\%) y otros (16\%). El contenido de resina se determinó por extracción de ácidos resínicos con diclorometano, por 5-6 h, según técnica TAPPI 204 om-88 (TAPPI, 1988). 
2. Las cepas nativas fueron aisladas a partir de pitch, de astilla y de pulpa de la planta PIPSA-MEX S.A. de C.V. Se utilizó la técnica de cultivo de enriquecimiento (Schlegel, 1997). Los microorganismos se incubaron en medio mineral líquido; como única fuente de carbono se utilizó pitch, astilla y pulpa de madera a temperatura ambiente durante 30 días a $100 \mathrm{rpm}$. Durante este tiempo, se efectuaron observaciones al microscopio para comprobar crecimiento. Después de 30 días de incubación $\left(28-30{ }^{\circ} \mathrm{C}\right)$ por enriquecimiento, se sembró una muestra de cada tratamiento en medio sólido adicionado con pitch por estría cruzada. Los cultivos se purificaron mediante la técnica de diluciones.

3. La observación macroscópica de cepas, se realizó sobre medios de cultivo sólido en caja Petri, analizando color, tamaño y aspecto de las colonias. En el caso de bacterias, se realizó la tinción diferencial de Gram (Schlegel, 1997). Para los hongos filamentosos, se aplicó la técnica de Microcultivo de Ridell (Ridell, 1951). Para la observación de levaduras se realizaron frotis con azul de lactofenol (Adame y Becerril,1998). Los cultivos puros fueron conservados en tubo inclinado con medio sólido adicionado con pitch, $\mathrm{a} 4^{\circ} \mathrm{C}$.

4. La identificación se complementó con un microscopio de alta definición. Se observaron los microcultivos de Ridell (previamente elaborados) para seguir el crecimiento del hongo, su esporulación, la forma de sus hifas y su tipo de esporas (Adame y Becerril, 1998).

5. La identificación de bacterias se realizó con el uso de Productos Analíticos para Identificación (API), combinada con algunas pruebas alternativas de oxidación, movilidad y oxido-fermentación. Los resultados obtenidos en las galerías y pruebas complementarias fueron transferidos a un Software de Identificación (APIAnalytical Profile Index) para conocer o confirmar la identidad de las cepas estudiadas.

6. El sistema de fermentación semisólida se desarrolló con Paecilomyces sp., seleccionado por su rendimiento en degradación de ácidos resínicos en astilla.

$1000 \mathrm{~g}$ de astilla en una charola rectangular $(30 \mathrm{~cm} \times 19 \mathrm{~cm} \times 8 \mathrm{~cm})$, se inocularon con una suspensión de esporas en medio mineral de Peacilomyces sp. ( $\mathrm{pH} 5,5$ 6). Cada $48 \mathrm{~h}$ el sistema fue aireado manualmente y mantenido a temperatura ambiente y $50 \%$ de humedad (Villegas et al., 1993). Después de 15 días, se realizó la cinética de degradación de ácidos resínicos para evaluar el tratamiento a escala planta piloto. Cada experimento se realizó por triplicado.

7. Los ensayos preliminares de heap leaching se realizaron con Chryseomonas luteola sobre $1000 \mathrm{~g}$ de astilla en un recipiente rectangular $(31 \mathrm{~cm} \times 23,5 \mathrm{~cm} \times 7,5$ $\mathrm{cm}$ ) con orificios de $0,5 \mathrm{~cm} \times 0,5 \mathrm{~cm} \times 0,5 \mathrm{~cm}$ en todas su caras y colocado a $5^{\circ}$ de inclinación (Medrano y Galán, 2000). Las astillas se inocularon con la suspensión bacteriana en solución mineral (1 L) asperjada con una pistola de aire (DeVilbiss MGQ-300) controlada para ofrecer un flujo aproximadamente constante de $1 \mathrm{ml} / \mathrm{min}$. La pistola se recargó cada 12 h con suspensión fresca y contabilizada, recolectando cada 48 $\mathrm{h}$ las aguas madres.

\section{RESULTADOS Y DISCUSIÓN}

El contenido inicial promedio de resinas en el lote evaluado fue de $43 \%$. Las repeticiones para cada tratamiento fueron por triplicado. Durante el proceso de aislamiento se obtuvieron 49 cepas diferentes. Estos cultivos puros crecieron sin ningún problema en medio sólido adicionado con pitch. De esta manera se descartó la presencia de cepas totalmente celulolíticas. La identificación preliminar de las 49 cepas aisladas dio por resultado: 13 bacterias, 18 hongos filamentosos y 18 levaduras (Tablas 1,2 y 3 ).

El análisis de variancia, con respecto a los extraíbles a los diferentes tiempos, indicó que todas las cepas produjeron una degradación de los ácidos resínicos pero con diferencias significativas. Un concentrado de estos rendimientos se muestra en la Tabla 4. 
Tabla 1. Cepas aisladas en cada tratamiento cuya fuente de carbono principal son los ácidos resínicos

\begin{tabular}{ccc}
\hline \multicolumn{2}{c}{ Pitch } & \\
\hline Pitch & Astilla & Pulpa \\
\hline 9 Levaduras & 8 Levaduras & 5 Bacterias \\
\hline 8 Bacterias & 7 Hongos filam entosos & 1 Levadura \\
\hline
\end{tabular}

11 Hongos filamentosos

Tabla 2. Identificación de bacterias aisladas según la tinción de Gram

\begin{tabular}{lll}
\hline $\begin{array}{l}\text { Cantidad de } \\
\text { cepas }\end{array}$ & GRAM. \\
\hline 7 Bacterias & Negativo & $(-)$ \\
\hline 6 Bacterias & Positivo & $(+)$ \\
\hline
\end{tabular}

Tabla 3. Identificación preliminar de hongos obtenidos de diferente fuente de aislamiento

\begin{tabular}{ll}
\multicolumn{1}{c}{ Pitch } & \multicolumn{1}{c}{ astilla } \\
\hline 4 Aspergillus & 1 Aspergillus \\
\hline 5 Penicillum & 3 Penicillum \\
\hline 1 Alternaria & \\
\hline 3 Desconocidos & \\
\hline
\end{tabular}


El tiempo es un factor que influye significativamente en la degradación de los ácidos resínicos en cada uno de los tratamientos.

La Figura 1 muestra el resultado del análisis de variancia con la técnica de Duncan. En esta gráfica se representa el promedio del contenido de extraíbles en cada tratamiento a dos tiempos. Las cepas $\mathrm{C} 1$ a C49 corresponden a los tratamientos biológicos a 15 días, mientras que las cepas C50 a C98 corresponden a los tratamientos biológicos a 30 días. Por ejemplo, la cepa $\mathrm{C} 1$ es la misma que la $\mathrm{C} 50$, sólo que el tratamiento es a tiempos diferentes, la cepa C2 es la misma que la 51 y así sucesivamente.

Se seleccionaron las cepas que degradaron la mayor cantidad posible de extraíbles. En particular se prefirieron aquellas cepas que dejaron un remanente de alrededor de $1 \%$ o menor de extraíbles. Se eligieron cuatro hongos $(\mathrm{H})$, dos bacterias $(B)$ y tres levaduras $(L)$. En la gráfica de la Figura 2 se visualiza la cinética de degradación de extraíbles que sigue un comportamiento similar en todos los tratamientos. Alos 15 días de tratamiento biológico se observa una evidente disminución de extraíbles.

\section{Fermentación semisólida}

El sistema se inoculó con una suspensión de esporas en medio mineral a una concentración de $2,175 \times 10^{10}$ esporas de Paecilomyces sp. por $\mathrm{mL}$. En la Tabla 5 se muestra la degradación de ácidos resínicos después del tratamiento de astilla por fermentación semisólida con Paecilomyces $\mathrm{sp}$.

Se obtuvo mayor degradación de pitch en matraz agitado. En este caso una degradación del $28,37 \%$ por tratarse de resultados preliminares es halagüeña, por lo que a futuro podría profundizarse y definir el efecto de la concentración de biomasa en el inóculo, así como aspectos de transferencia de masa y calor que puedan incrementar la eficiencia de la degradación (Ryoo et al., 1991). Por otro parte, dado el tipo de fermentación, el perfil de distribución de

Tabla 4. Degradación de pitch por los microorganismos seleccionados

\begin{tabular}{clccc}
\hline Cepa (clave) & Microorganismo & \multicolumn{2}{c}{ \% de resina* } & \% de eliminación \\
\cline { 3 - 5 } & & 15 dias & 30 días & \\
\hline H1 (C1/C50) & Paecilomyces sp. & 0,7986 & 0,5747 & 86,63 \\
\hline H2 (C39/C88) & Penicillum sp. & 1,1806 & 1,1547 & 73,14 \\
\hline H3 (C42/C91) & Penicillum sp. & 0,7060 & 0,5882 & 86,32 \\
\hline H4 (C11/C60) & Phialophora sp. & 1,3562 & 0,7969 & 81,46 \\
\hline B1 (C38/C87) & Chryseomonas luteola 0,5837 & 0,5447 & 87,33 \\
\hline B2 (C23/C72) & Xanthomonas sp. & 1,2158 & 0,7849 & 81,74 \\
\hline L1 (C44/C93) & Rhodothorula sp. & 1,1592 & 0,9816 & 77,17 \\
\hline L2 (C36/C85) & Cryptococcus sp. & 1,1199 & 1,0473 & 75,64 \\
\hline L3 (C15/C64) & Trichosporon sp. & 1,2491 & 0,5890 & 86,30 \\
\hline
\end{tabular}

* Contenido inicial de resina: 4,3\% 


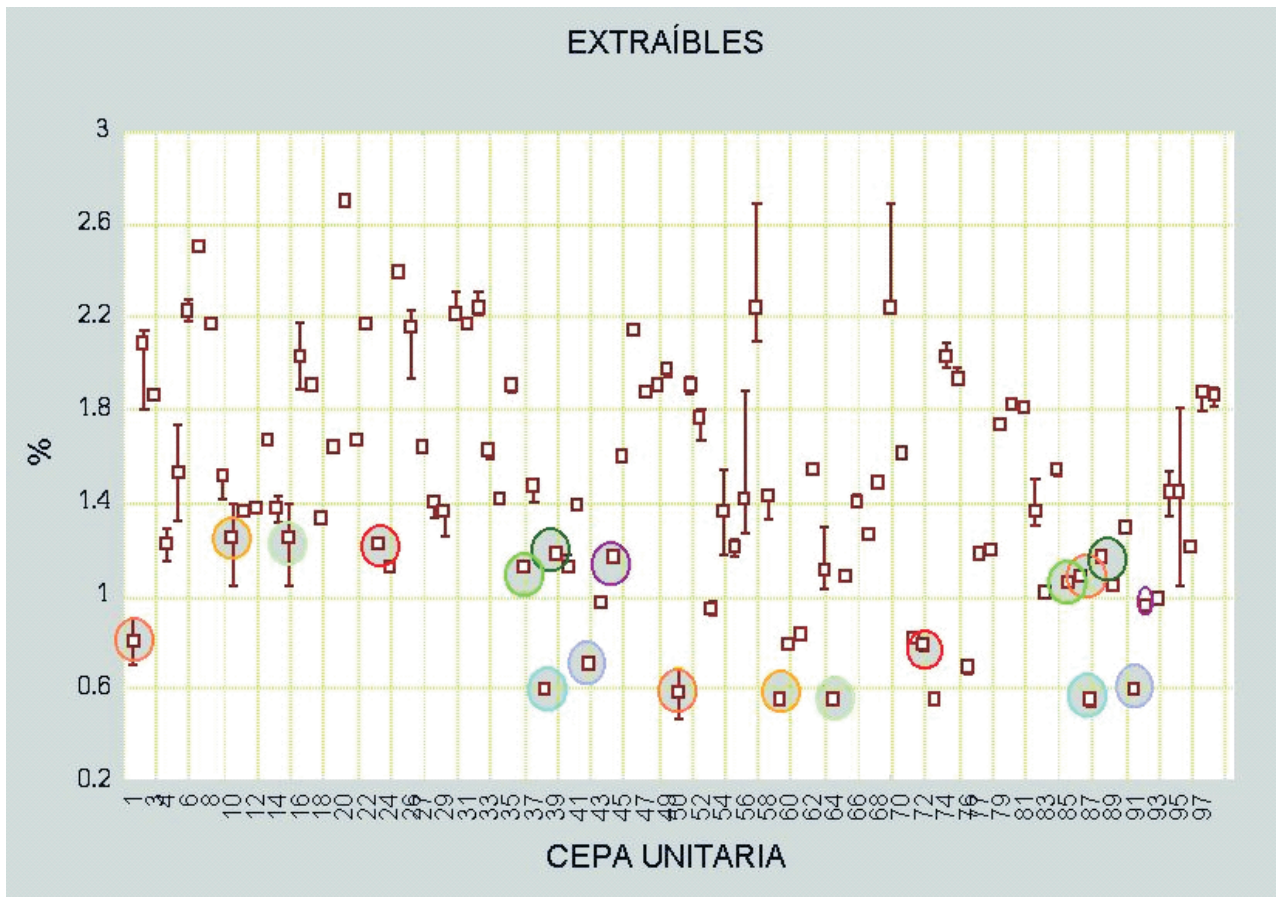

Figura 1. Gráfica del porcentaje de extraíbles a diferente tiempo de tratamiento biológico de la muestra por cada cepa aislada.

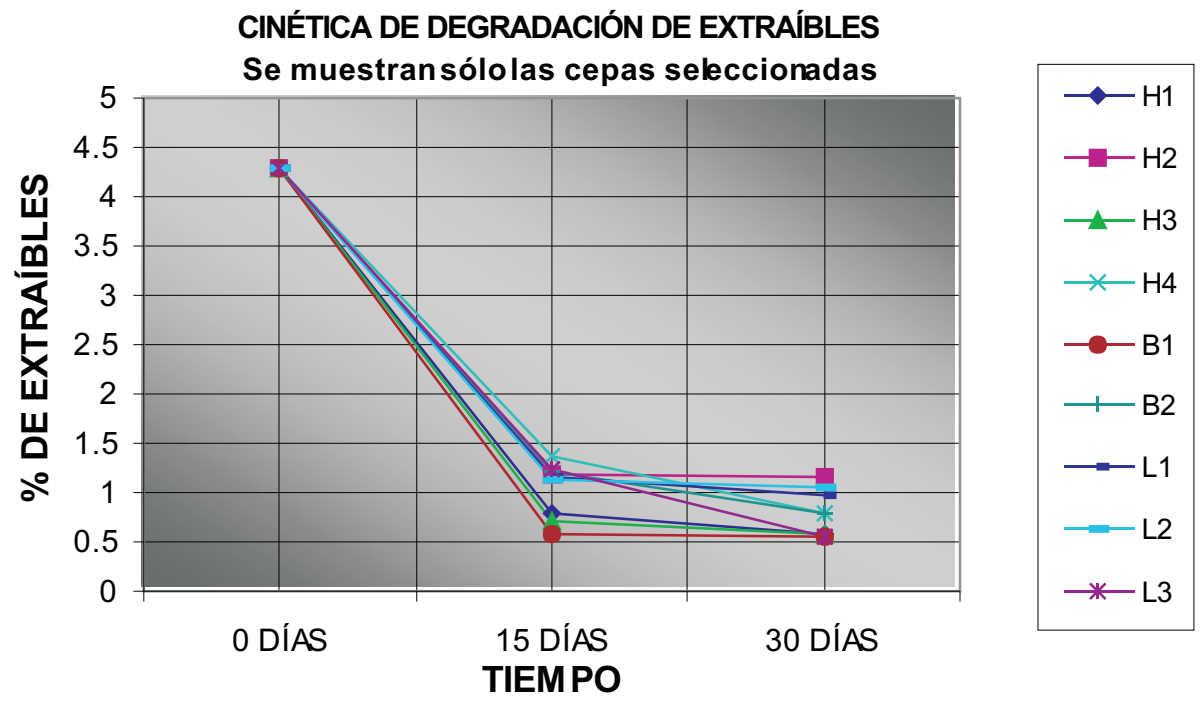

Figura 2. Cinética de degradación de extraíbles por cada cepa unitaria, a un tiempo cero, 15 y 30 días. 
Tabla 5. Degradación de pitch en matraz agitado y en fermentación semi-sólida

\begin{tabular}{cc}
\hline \multicolumn{2}{c}{$\begin{array}{c}\text { Degradación de pitch a } \\
15 \text { días de tratamiento } \\
\text { biológico }\end{array}$} \\
\hline \begin{tabular}{cc} 
Matraz agitado & Fermentación semi-sólida \\
\hline $86,63 \%$ & $28,37 \%$ \\
\hline
\end{tabular}
\end{tabular}

esporas en el material será de gran importancia para obtener resultados más significativos (Silman, 1980). La oxigenación es otro de los papeles importantes en este tipo de procesos, por lo que su cuantificación deberá visualizarse también (Durand et al., 1988). Los factores mencionados son de gran importancia para promover la formación de un sistema enzimático más eficaz.

\section{Heap leaching}

La concentración promedio de la suspensión bacteriana en solución mineral de Chryseomonas luteola que fue asperjada sobre las astillas cada $12 \mathrm{~h}$ fue de $4 \times 10^{10}$ bacterias $/ \mathrm{mL}$. La concentración promedio del inóculo de solución recirculada fue de $9 \times 10^{13}$ bacterias $/ \mathrm{mL}$. La Tabla 6 muestra los resultados de la degradación de ácidos resínicos con el tratamiento biológico de la astilla en heap leaching con Chryseomonas luteola.

Al comparar los resultados de la fermentación semi-sólida y heap leaching, se encuentra que en esta última, donde se logró una degradación pobre de pitch, se puede establecer que la velocidad de flujo no permitió un perfil de distribución adecuado de microorganismos, como lo fueron las esporas en la fermentación semi-sólida. Sin embargo, el resultado puede ser prometedor si en futuros trabajos se experimenta en un sistema combinado, es decir, heap-leachingsemisólido (Gibbon et al., 1986). Bajo este criterio, podría establecerse un sistema de recirculación intermitente, que a otros investigadores les ha dado mejores resultados (Lonsane y Ahmed,1989). En este experimento se percibe también que el consumo de energía para una bomba de recirculación sería de importancia económica (Osterhuis y Kossen,1981). La gran ventaja de este sistema sería la generación de una metodología relativamente sencilla para su escalamiento (Oldshue, 1966).

\section{CONCLUSIONES}

Con base en los resultados obtenidos en el presente trabajo se puede afirmar que en principio el proceso biotecnológico para eliminar ácidos resínicos precursores de pitch en astilla de madera de pino es viable ya que:

1. Se lograron aislar 49 cepas nativas degradadoras de ácidos resínicos a partir de pitch, astilla y pulpa de madera.

2. Después de los tratamientos biológicos se obtuvieron abatimientos significativos en el contenido inicial de ácidos resínicos $(4,3 \%)$.

3. En la cinética de degradación de ácidos resínicos se muestra que todas las cepas tienen la misma tendencia de degradación de estos compuestos. De hecho, a los 15 días hay una disminución significativa en la mayoría de los casos.

4. Se seleccionaron 9 cepas diferentes: 4 de hongos filamentosos, 2 de bacterias y 2 de levaduras, como las mejores cepas degradadoras de ácidos resínicos.

5. Se logró identificar el género de 7 cepas seleccionadas: Paecilomyces sp., 
Tabla 6. Comparación de degradación de pitch en matraz agitado y en heap leaching

\begin{tabular}{cc}
\hline $\begin{array}{c}\text { Degradación de pitch a } \\
15 \text { días de tratamiento } \\
\text { biológico }\end{array}$ \\
\hline Matraz agitado & Heap leaching \\
\hline $87,33 \%$ & $10,84 \%$ \\
\hline
\end{tabular}

Penicillum sp., Penicillum sp., Phialophora sp., Trichosporon sp., Rhodotorula sp. y Cryptococcus sp. En una cepa se identificó también la especie Chryseomonas luteola y otra no se pudo identificar.

6. La cepa Chryseomonas luteola fue la más eficiente para la degradación de ácidos resínicos con un 87,33\% de efectividad, y fue utilizada para llevar a cabo los ensayos preliminares del proceso heap leaching.

7. La cepa Paecilomyces sp. ocupó el segundo lugar con un $86,63 \%$ de efectividad en la degradación de ácidos resínicos, y se le utilizó para llevar a cabo los ensayos preliminares del proceso fermentación semisólida.

8. Sin embargo, en los ensayos preliminares no se tuvieron los mismos resultados de degradación de ácidos resínicos como los obtenidos en los tratamientos a nivel matraz. En el heap leaching, el porcentaje de eliminación de ácidos resínicos por Chryseomonas luteola a un tiempo de 15 días fue de $10,84 \%$, resultado por debajo del obtenido a nivel matraz, mientras que en la fermentación semi-sólida el porcentaje de eliminación por Paecilomyces sp. al mismo tiempo fue de $28,37 \%$, también muy bajo comparado con el obtenido a nivel matraz.

Por lo anterior, se recomienda continuar la identificación de las cepas seleccionadas y aspirar a manipularlas genéticamente para mejorar los resultados obtenidos en este estudio. Asimismo, en la fermentación semisólida, probar otras concentraciones de inóculo, tiempos de incubación y aireación para aproximarse a los resultados obtenidos a nivel matraz. En el proceso de heap leaching, experimentar con otras concentraciones de inóculo, varias velocidades de flujo, así como el tiempo de incubación. Desafortunadamente, por razones de mercado principalmente, la empresa ha cerrado indefinidamente sus puertas, posponiendo para mejor ocasión la aplicación in situ de los resultados y recomendaciones derivados de este trabajo.

\section{RECONOCIMIENTOS}

Este proyecto fue realizado en colaboración con la empresa PIPSA-MEX y con apoyo financiero del Fideicomiso FOSIVILLA-CONACYT, según convenio 20000405007

\section{REFERENCIAS}

Adame, J.M. y P. Becerril, 1998. Manual de Prácticas, Laboratorio de Micología Médica. UANL, FCB, Departamento de Microbiología.

Aguilar Rivera, N. 2004. Importancia, manejo y control de extraíbles e incrustaciones (pitch) en la fabricación de papel. Madera y Bosques 10(1): 89-99

Back, L.E. y L. H. Allen, 2000. Pitch Control, 
Wood Resin and Deresination. TAPPI PRESS.

Brush, T.S., R.L. Farell, y C. Ho, 1994. Biodegradation of wood extractives from southern yellow pine by Ophiostoma piliferum. Tappi Journal 77(1):155.

Durand, A., P. Pichol, y C. Desgranges, 1988. Approaches to $K_{\mathrm{La}}$ measurements in solid state fermentations: Biotechnology Techniques 2:11.

Gibbon, W.R., C. A. Westby, y T. L. Dobbs, 1986. Intermediate scale semicontinuous solid phase fermentation process for production of fuel ethanol from sweet sorghum. Appl. Environmental Microbiology. 51:115.

Lonsane, B.K. y S.Y. Ahmed,1989. Reduction, recycle, utilization and exchange. En Souvenir, National Symposium on Impact of Pollution in and from Food Industries and its Management. Association of Food Scientist and Technologist. Mysore, India. pp 33.

Lloyd, J.A., N.A. Deacon, y C.W. Horne, 1990. Resin during process TMP y CTMP. Journal Appita 43(6):429.

Medrano, R.H. y W.L. Galán, 2000. Biotecnología de Minerales. CONACYTCOCYTED. México.

Mitsui, M., H. Taneda., Y Fujita., M. Matsukara., y K. Hata. 1998. Biodegradation of Resin Acids in the Papermaking Process. Seventh International Biotechnology in the Pulp and Paper Industry Conference Proceedings, CPPA, Vancouver, B.C. Canada. 217.

Oldshue, J.Y. 1966. Fermentation mixing scale-up techniques. Biotechnology Bioeng. 18:3.

Manuscrito recibido el 17 de febrero de 2005. Aceptado el 7 de Junio de 2005.

Este documento se debe citar como:

Rodríguez, C. , H. Medrano, N. Rocha, A. Gallegos, M. Rosales y R. González-Laredo. Tratamiento biológico de madera para eliminar pitch en la producción de celulosa. Madera y Bosques 11(1): 19-27.
Osterhuis, N.M.G. y N.W.F. Kossen, 1981. Power input measurements in a production scale bioreactor. Biotechnology Letts. 3:645.

Ridell, R.W. 1951. Survey of Fungus Diseases in Britain. Br Med Bull. 7:197.

Rocheleau, M.-J., B.B. Sitholé., L.H. Allen, y Y.Noel, 1997. Fungal Treatment of Chips for Pitch Control: Effect of Aged Aspen at Room Temperature and at $5^{\circ} \mathrm{C}$. Ninth International Wood and Pulping Chemistry Conference proceedings, CPPA, Montreal Canada. pp 96.

Ryoo, D., V.G. Murphy., N.M. Karim, y R.P.Tengerdy, 1991. Evaporative temperature and moisture control in a rocking reactor for solid substrate fermentation. Biotechnology Techniques. 5:19.

Schlegel, H.G. 1997. General Microbiology. Seventh Edition, Cambridge University Press.

Silman, R.W. 1980. Enzyme formation during solid substrate fermentation in rotating vessels. Biotechnology Bioeng. 22:411.

TAPPI 204 om-88.1988. Extractivos de madera y pulpa. TAPPIPRESS.

TAPPI 257 cm-85. 1985. Muestreo y Preparación de madera para análisis. TAPPIPRESS.

Villegas, E., Aubague, S., Alcantara, L., Auria, R. y Revah, S. 1993. Solid State Fermentation: Acid Protease Production in Controlled $\mathrm{CO}_{2}$ and $\mathrm{O}_{2}$ Enviroments. En Biotechnology Advances, Research Reviews and Patent Abstracts. MooYoung, E., Glick, B.R. y Bols, N.B. Eds. Pergamon press. pp 387. 